\title{
Stratospheric Multiple Equilibria and Seasonal Variations
}

\author{
By Y. Wakata and M. Uryu \\ Department of Physics, Faculty of Science, Kyushu University, Fukuoka 812, Japan \\ (Manuscript received 4 September 1986, in revised form 8 December, 1986)
}

\begin{abstract}
Using a vertical one-dimensional model, we discuss the equilibrium of the stratospheric mean zonal flow and planetary wave forced by the surface corrugation. Their stabilities are also examined.

It is shown that in late autumn and early spring, there exist two stable equilibrium solutions and one unstable equilibrium solution, while one stable equilibrium solution in mid-winter. It is noted that we have no vacillating solution as obtained by Holton and Mass (1976) and Chao (1985).

Solving the time-dependent problems with changing the vertical shear of the basic zonal wind with one year period as an indicator of seasonal variation, we can obtain the growth of planetary wave twice in winter and the intensification of westerly wind between these two amplitude peaks. In addition, it is shown that a drastic transition of the mean zonal winds from westerlies to easterlies resembling the final warming occurs in later winter or early spring. These are very similar to the results of data analysis by Smith (1983) and those of the numerical simulation by Holton and Wehrbein (1980). Thus, the stratospheric seasonal variation can be interpreted as a hysteresis phenomenon caused by the change in the solar differential heating in the presence of multiple equilibria:

The breakdown of polar. night vortex with the final warming is a kind of nonlinear instability. That is, if the winter westerly wind in the upper stratosphere is close to the Charney-Drazin's critical velocity and if it is perturbed by a planetary wave, then the refractive index is changed and this change causes the amplification of the wave. The amplified wave, in turn, changes the refractive index is changed and this change causes the amplification of the wave. The amplified wave, in turn, changes the refractive index by accelerating easterlies. This process provides a feedback, and the polar night vortex breaks down in the end. The present instability is not the resonance breakdown as in Plumb (1981).

Finally, we make some comments on Chao's (1985) results.
\end{abstract}

\section{Introduction}

The stratospheric zonal mean winds with westerlies in winter and easterlies in summer are caused basically by the solar differential heating and also affected by planetary waves propagating from the troposphere.

The easterlies are gradually taken over by westerlies in autumn. In contrast to this, the westerlies change drastically into the easterlies in late winter or early spring, with warming in the polar region. This is called final warming. The sudden warming phenomena repeat ordinarily a few times until "permanent" easterlies appear. However, the main concerns in the present paper are not focused on each warming

() 1987, Meteorological Society of Japan event but on rather long-term behaviors, the growth of planetary wave occurring twice in winter, the intensification of the mean zonal wind between these two wave amplitudes peaks and the final drastic breakdown of the polar vortex in early spring (Smith, 1983; Holton and Wehrbein, 1980). The sudden warming is now believed to be one of the most notable phenomena which are manifested as a result of the interaction between planetary waves and the mean zonal flows, since Matsuno (1971) who succeeded in simulating the main features of the phenomenon by including the easterly acceleration effect of upwasrd propagating planetary wave and the resulting critical layer interaction. However, there remain, at least, two unresolved problems. One is why the planetary 
wave can be amplified in the upper troposphere as assumed in Matsuno's model, the other is why the sudden warmings prefer late winter or early spring to other seasons.

As to the first problem, Clark (1974) and Tung and Lindzen (1979a, b) proposed the linear resonance of planetary wave as the amplification mechanism, while Plumb (1981) assumed the nonlinear resonance of planetary wave in the stratosphere by assuming that the wave has a fixed shape in the vertical as well as in the horizontal direction, and discussed te so-called topographic instability of the finite amplitude wave, leading to the breakdown of polar vortex with warming of the polar stratosphere. As Plumb's model does not include any dissipation process, the wave repeats its growth and decay alternately. If some dissipation such as Newtonian cooling and Ekman damping is incorporated, multiple equilibrium (three) solutions can be obtained as shown by, for example, Wakata and Uryu (1984a), and also by Charney and DeVore (1979) based on the highly truncated, one layer spectral model. The topographic instability belongs essentially to the middle branch instability of cusp type catastrophe. Then, we may ask whether there exist any multiple equilibria if nonlinear effects together with dissipation are important in the sudden warming phenomena, and how an atmosphere in multiple equilibria responds to the change in the external parameter such as the solar differential heating.

On the other hand, Palmer (1981a, b), O'Neill and Youngblut (1982) and Kanzawa (1982) analyzed the satellite data and showed that just before the sudden warming, the distribution of mean zonal winds has larger refractive index in the high latitudinal region than as usual, and that planetary waves which propagate southward in ordinary situation are directed northward. Such an extraordinary configuration of the mean zonal winds could be helpful to the growth of planetary wave. However, we have a question here, the second question above, why the mean zonal winds have such a configuration in that season, in spite that the external parameter such as the solar differential heating is same in late autumn and early spring. The observed configuration could be, at least partly, resulted from the nonlinear interaction between the mean zonal wind and planetary waves.

Thus, we will treat a nonlinear system in which planetary waves and the mean zonal wind affect mutually, and its response to the external forcing which changes in time, i.e., seasonally. For simplicity, we use a vertical one-dimensional model adopted by Geisler (1974) for simulating the sudden warming produced by the topographic forcing. It is noted here that by solving the initial value problems with Holton and Mass' model (1976). Chao (1985) has discussed the sudden warming in the light of the concept of catastrophe. Obtaining the marginal curve between the steady and the vacillating regimes by solving the time-evolution problem with changing the bottom geopotential height, he pointed out that the steady wave regime and the vacillation regime obtained by Holton and Mass (ibid) could be multiple equilibrium solutions for a given set of the external parameters. However, we note here that the full equilibrium solutions cannot be obtained and their stabilities cannot be checked by such a method as Chao's. Thus, he has not fully discussed the nonlinear characteristics of the model, although it must be important to examine how many equilibrium solutions can exist and how the model atmosphere with such multiple steady states respond to the change in seasonal parameters. In addition, it should not be overlooked that he as well as Helton and Mass (ibid) set geopotential height at the bottom. This condition is not appropriate to the topographic forcing problem, becaue the free modes with zero vertical velocity at the bottom and hence the barotropic resonance cannot be suitably expressed by this condition. In our model, the vertical velocity is given at the bottom. We shall briefly mention the stability properties of Chao's or Holton and Mass' model in Appendix B.

In section 2, our model and the basic equations are mentioned. Section 3 treats briefly the linear response for the sake of later discussion on the nonlinear problem. The nonlinear equilibrium solutions and their stabilities are discussed in section 4 , and we see that multiple equilibria appear in late autumn and late winter season. The solution for time-dependent problem is mentioned in section 5 , to see that planetary waves grow twice in winter and strong westerlies 
appear between these two amplitude peaks and a drastic change from westerlies to easterlies resembling the final warming phenomenon occurs in late winter or early spring. It is noted that these results are very similar to those obtained by Smith (1983) based on the data analysis of observations and also by Holton and Wehrbein (1983) with use of a numerical model. The final section is devoted to the discussion and conclusion.

\section{Model and the basic equations}

In order to investigate nonlinear interaction between planetary wave and the mean zonal flow, we adopt the following quasi-geostrophic potential vorticity equation in log-pressure coordinate on a $\beta$-plane centered at $45^{\circ}$ (cf. Holton, 1975; Wataka and Uryu, 1984a), with use of the notations listed in Appendix A:

$$
\begin{aligned}
\left(\frac{\partial}{\partial t}+\right. & +\boldsymbol{u} \cdot \nabla)\left(\nabla^{2}+\frac{\sin ^{2} \phi_{0}}{B r}\left(\frac{\partial}{\partial z^{2}}-\frac{1}{4}\right)\right) \Phi+\beta \Phi_{x} \\
= & -\alpha_{R} \nabla^{2} \Phi-\frac{\sin ^{2} \phi_{0}}{B r}\left(\alpha_{N}\left(\frac{\partial}{\partial z^{2}}-\frac{1}{4}\right)\right. \\
& \left.+\frac{\partial \alpha_{N}}{\partial z}\left(\frac{\partial}{\partial z}+\frac{1}{2}\right)\right) \Phi \ldots \ldots \ldots \ldots \ldots(1)
\end{aligned}
$$

where all the quantities are made dimensionless by the scale-unit presented also in Appendix A.

The geostrophic and hydrostatic relations are expressed,

$$
\begin{aligned}
& \boldsymbol{k} \times \boldsymbol{u}=-e^{2 / 2} \nabla \Phi \ldots \ldots \\
& T=\sin \phi_{0} \frac{\partial}{\partial z}\left(e^{2 / 2} \Phi\right)
\end{aligned}
$$

As the bottom boundary condition, we assume the Ekman pumping in addition to the topographic forcing:

$$
\hat{w}=\boldsymbol{u} \cdot \nabla h+\hat{E}^{1 / 2} \nabla^{2} \Phi \quad \text { at } z=0
$$

Utilizing the relation between the real vertical velocity and the vertical 'velocity' in the log-pressure coordinate together with the thermodynamics equation, we can rewrite the condition (4) in terms of $\Phi$ as follows:

$$
\begin{gathered}
\left(\frac{\partial}{\partial t}+\boldsymbol{u} \cdot \nabla\right)\left(\frac{\partial}{\partial z}+\left(\frac{1}{2}-S\right)\right) \Phi \\
+\alpha_{N}\left(\frac{\partial}{\partial z}+\frac{1}{2}\right) \Phi
\end{gathered}
$$

$$
=-\boldsymbol{u} \nabla \hat{h}-E^{1 / 2} \nabla^{2} \Phi
$$

where $\hat{h}=B r /\left(\operatorname{Rosin} \phi_{0}\right) h$ and $E^{1 / 2}=\hat{E}^{1 / 2} B r /$ $\left(R o \sin \phi_{0}\right)$. In what follows, we assume the following sinusoidal form for the bottom corrugation:

$$
\hat{h}=h_{0} \sin l y e^{i k x}+\text { comp. conj. }
$$

Following Geisler (ibid) and Holton and Msss (ibid), we assume that geopotential $\Phi$ consists of zonal flow and one wave component with zonal wave number $k$ and with only one meridional mode as follows.

$$
\begin{aligned}
\Phi= & \frac{1}{l}\left(U_{R} e^{-z / 2}+\bar{u}(t, z)\right) \cos l y \\
& +\left(\phi(t, z) e^{i k x}+\text { comp. conj. }\right) \sin l y \cdots
\end{aligned}
$$

where $U_{R}$ is the zonal flow due to the meridional temperature gradient caused by the solar differential heating and $\bar{u}$ is the wave induced mean zonal flow. In the following, we use asterisk $(*)$ to represent dimensional quantities.

$U_{R}^{*}$, the dimensional form of $U_{R}$, is assumed to be

$$
U_{R}^{*}=10+\Lambda z^{*}(\mathrm{~m} / \mathrm{s})
$$

where $z^{*}$ is height in $\mathrm{km}$ unit. Parameter $\Lambda$ is the vertical shear due to the meridional temperature gradient. It is noted that $\Lambda$ is related numerically to the meridional temperature difference over the distance $5000 \mathrm{~km}$ as $\Delta T^{*} \sim$ $10 \cdot \Lambda(K)$, which is obtained from the thermal wind relation. Thus, the seasonal change in the basic temperature distribution can be represented by changing $\Lambda$. In section 5 , we will discuss the nonlinear response of our model atmosphere to the change of $\Lambda$ with one year period. We assume a sponge layer from altitude $80 \mathrm{~km}$ to $110 \mathrm{~km}$ for avoiding the reflection from the top boundary, where the basic zonal flow $U_{R}^{*}$ is assumed to be independent of height with magnitude $10+90 \Lambda(\mathrm{m} / \mathrm{s})$ and to connect smoothly with the lower winds. Further, in the sponge layer, we assume Rayleigh friction in the wave equation, of which coefficient increases from zero to decay time of 1 day with height. In the lower layers, the Rayleigh friction is not included.

The Newtonian cooling coefficient is assumed as 


$$
\alpha_{N}^{*}=\left\{1.5+\tanh \left(\left(z^{*}-35\right) / 7\right)\right\} \times 10^{-6}\left(s^{-1}\right)
$$

which is the same as that adopted by Holton and Mass (ibid). The corresponding damping time is 20 days in the troposphere and 5 days above 50 $\mathrm{km}$.

The Ekman number $E^{1 / 2}$ is set at 0.0402 with an eddy viscosity of $8 \mathrm{~m}^{2} / \mathrm{s}$. The amplitude of the bottom forcing $h_{0}$ is assumed to be $50(\mathrm{~m})$ unless otherwise mentioned. The corresponding height of mountain is $100 \mathrm{~m}$ by the definition (6). Wave numbers $k$ and $l$ are set at $k=2.21\left(k^{*}=2 / a^{*} \cos (\pi)\right.$ 2) and $l=\pi$.

Substituting $\Phi$ from (7) into (1), multiplying $\sin l y$ and integrating in the meridional direction, we obtain the equations for the real part $\phi_{R}$ and the imaginary part $\phi_{I}$ of wave disturbance $\phi$ as follows:

$$
\begin{aligned}
\frac{\partial}{\partial t}[- & \left.\left(k^{2}+l^{2}\right)+\frac{\sin ^{2} \phi_{0}}{B r}\left(\frac{\partial^{2}}{\partial z^{2}}-\frac{1}{4}\right)\right] \phi_{R} \\
= & q k \varepsilon\left(U_{R}+\bar{u} e^{z / 2}\right) \\
& {\left[-\left(k^{2}+l^{2}\right) \phi_{I}+\frac{\sin ^{2} \phi_{0}}{B r}\left(\frac{\partial^{2}}{\partial z^{2}}-\frac{1}{4}\right) \phi_{I}\right] } \\
& +q k \phi_{I}\left[\beta+e^{z / 2} \varepsilon\left(l^{2}-\frac{\sin ^{2} \phi_{0}}{B r}\left(\frac{\partial^{2}}{\partial z^{2}}-\frac{1}{4}\right)\right)\right. \\
& \left.\cdot\left(U_{k} e^{-z / 2}+\bar{u}\right)\right]-\frac{\sin ^{2} \phi_{0}}{B r}\left[\alpha_{N}\left(\frac{\partial^{2}}{\partial z^{2}}-\frac{1}{4}\right)\right. \\
& \left.+\frac{\partial \alpha_{N}}{\partial z}\left(\frac{\partial}{\partial z}+\frac{1}{2}\right)\right] \phi_{R}+\left(k^{2}+l^{2}\right) \alpha_{R} \phi_{R}
\end{aligned}
$$

with the boundary conditions

$$
\begin{aligned}
\frac{\partial}{\partial t}\left[\frac{\partial}{\partial z}+\frac{1}{2}-S\right] \phi_{R} \\
=q k \varepsilon\left(U_{R}+\bar{u}\right)\left[\frac{\partial}{\partial z}+\frac{1}{2}-S\right] \phi_{I} \\
\quad-q k \varepsilon \phi_{I}\left[\frac{\partial}{\partial z}+\frac{1}{2}-S\right]\left(U_{R} e^{-z / 2}+\bar{u}\right) \\
\quad-\alpha_{N}\left(\frac{\partial}{\partial z}+\frac{1}{2}\right) \phi_{R}+E^{1 / 2}\left(k^{2}+l^{2}\right) \phi_{R} \\
\quad+\varepsilon\left(U_{R}+\bar{u}\right) k h_{0}\left(\frac{q-1}{2}\right) \text { at } z=0
\end{aligned}
$$

and

$\phi_{R}=0 \quad$ at $z=z_{T}$

where $\varepsilon=8 / 3 \pi$ and $q=1$. The equation and the boundary conditions for $\phi_{I}$ are readily obtained by replacing suffix $R$ by $I$ and setting $q=-1$ in the above equations, and are not shown here.

The equation and the boundary conditions for mean zonal flow can be obtained by the same procedure as for the wave equation except multiplying cos ly;

$$
\begin{aligned}
& \frac{\partial}{\partial t}[\left.-l^{2}+\frac{\sin ^{2} \phi_{0}}{B r}\left(\frac{\partial^{2}}{\partial z^{2}}-\frac{1}{4}\right)\right] \bar{u} \\
&=-\frac{\sin ^{2} \phi_{0}}{B r}\left[\alpha_{N}\left(\frac{\partial^{2}}{\partial z^{2}}-\frac{1}{4}\right)\right. \\
&\left.+\frac{\partial \alpha_{N}}{\partial z}\left(\frac{\partial}{\partial z}+\frac{1}{2}\right)\right] \bar{u}+2 \varepsilon k l^{2} \frac{\sin ^{2} \phi_{0}}{B r} \\
& \cdot e^{z / 2}\left(-\phi_{R} \frac{\partial^{2}}{\partial z^{2}} \phi_{I}+\phi_{I} \frac{\partial^{2}}{\partial z^{2}} \phi_{R}\right) \cdots \cdots(13) \\
& \frac{\partial}{\partial z}\left(\bar{u} e^{z / 2}\right)=0 \quad \text { at } z=z_{T} \cdots \cdots \cdots \cdots \cdots \cdots(14) \\
& \frac{\partial}{\partial t}\left[\frac{\partial}{\partial z}+\left(\frac{1}{2}-S\right)\right] \bar{u} \\
&=E^{1 / 2} l^{2} \bar{u}+2 k l^{2} \varepsilon\left(\phi_{I} \frac{\partial}{\partial z} \phi_{R}-\phi_{R} \frac{\partial}{\partial z} \phi_{I}\right) \\
& \quad+2 k l^{2} \varepsilon \phi_{I} h_{0}-\alpha_{N}\left(\frac{\partial}{\partial z}+\frac{1}{2}\right) \bar{u} \\
& \text { at } z=0 \cdots \cdots \cdots \cdots(15)
\end{aligned}
$$

We note here that wave-topography coupling term is included in the boundary condition (15).

In order to solve these equations, the vertical domain is represented by the $N$ grid points, of which the first one is under the ground and the $N$ th point at the top. In the present computations we set $N=24$, and hence the vertical grid distance is $5 \mathrm{~km}$. We transform the equations (10) to (15) including those for $\phi_{I}$ into the finite difference version, approximating the vertical derivatives by the centered difference. Then, we obtain the time evolution equations for $3 \mathrm{~N}$ unknown variables $\phi_{R}^{(j)}, \phi_{I}^{(j)}, \bar{u}^{(j)}$ and $(j=1,2, \ldots, N)$, which are written compactly as follows. 


$$
\frac{d}{d t} L\left(\begin{array}{c}
\phi_{R}^{(1)} \\
\phi_{R}^{(2)} \\
\vdots \\
\phi_{I}^{(1)} \\
\vdots \\
\bar{u}^{(N)}
\end{array}\right)=\left(\begin{array}{l}
f^{(1)}\left(\phi_{R}^{(1)}, \phi_{R}^{(2)}, \cdots, \bar{u}^{(N)}\right) \\
f^{(2)}\left(\phi_{R}^{(1)}, \phi_{R}^{(2)}, \cdots, \bar{u}^{(N)}\right) \\
f^{(N+1)}\left(\phi_{R}^{(1)}, \dot{\phi}_{R}^{(2)}, \cdots, \bar{u}^{(N)}\right) \\
\vdots \\
f^{(3 N)}\left(\phi_{R}^{(1)}, \phi_{R}^{(2)}, \cdots, \bar{u}^{(N)}\right)
\end{array}\right)
$$

where $L$ on the left-hand side is the coefficient matrix which is tri-diagonal except a few rows or columns including the boundary conditions, and the right-hand side consists of considerably complicated functions of $\phi_{R}^{(j)}, \phi_{I}^{(j)}$ and $\bar{u}^{(j)}$. Neither is shown here.

The equilibrium solutions can be obtained by setting the left-hand side to zero: the resulting nonlinear algebraic equations with $3 \mathrm{~N}$ degrees of freedom are solved by the Brent method ${ }^{(*)}$.

In order to examine the local stabilities of the equilibrium solutions, we use the perturbation method. That is, solution $\left(\phi_{R}^{(j)}, \phi_{I}^{(j)}, \bar{u}^{(j)}\right)$ is divided into the equilibrium solution and the time-dependent perturbation as follows.

$$
\left(\begin{array}{c}
\phi_{R}^{(1)} \\
\phi_{R}^{(2)} \\
\vdots \\
\phi_{I}^{(1)} \\
\vdots \\
\bar{u}^{(N)}
\end{array}\right)=\left(\begin{array}{c}
\phi_{R, S}^{(1)} \\
\phi_{R, S}^{(2)} \\
\vdots \\
\phi_{I, S}^{(1)} \\
\vdots \\
\left.\bar{u}_{, S}^{\prime \prime}\right)
\end{array}\right)+\left(\begin{array}{c}
\phi_{R}^{(1) \prime}(T) \\
\phi_{R}^{(2)^{\prime}}(T) \\
\vdots \\
\phi_{I}^{(1)^{\prime}}(T) \\
\vdots \\
\bar{u}^{N^{\prime}}(T)
\end{array}\right)
$$

where suffix $s$ and the prime indicate the equilibrium solution and the perturbation, respectively.

Substituting (17) into (16) and assuming that the perturbations are small around the equilibrium state, we retain the first order terms (cf. Wakata and Uryu, 1984b):

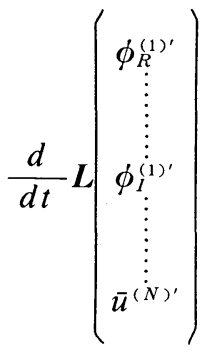

(*) See FACOM scientific subroutine library (SSL II).

$$
=\left(\begin{array}{cccc}
\frac{\partial f^{(1)}}{\partial \phi_{R}^{(1)}} & \frac{\partial f_{R}^{(1)}}{\partial \phi_{R}^{(2)}} & \cdots & \frac{\partial f^{(1)}}{\partial \bar{u}^{(N)}} \\
\vdots & \vdots & & \vdots \\
\frac{\partial f^{(N+1)}}{\partial \phi_{R}^{(1)}} & \frac{\partial f^{(N+1)}}{\partial \phi_{R}^{(2)}} & \cdots & \frac{\partial f^{(N+1)}}{\partial \bar{u}^{(N)}} \\
\vdots & \cdots & & \vdots \\
\frac{\partial f^{(3 N)}}{\partial \phi_{R}^{(1)}} & \frac{\partial f^{(3 N)}}{\partial \phi_{R}^{(2)}} & \cdots & \frac{\partial f^{(3 N)}}{\partial \bar{u}^{(N)}}
\end{array}\right)_{s}\left(\begin{array}{c}
\phi_{R}^{(1)^{\prime}} \\
\vdots \\
\vdots \\
\vdots \\
\vdots \\
\vdots \\
\bar{u}^{(1)^{\prime}} \\
\vdots \\
\end{array}\right)
$$

where the suffix $s$ attached to the matrix means that all the derivatives are evaluated at the equilibrium state. This equation is symbolically rewritten as

$$
\frac{d}{d t} \boldsymbol{L} \boldsymbol{X}=\boldsymbol{M} \boldsymbol{X}
$$

where $\boldsymbol{X}$ is the solution vector and $\mathbf{M}$ is the coefficient matrix.

Assuming that $X \sim X e^{\sigma t}$ with $\sigma=\sigma_{r}+i \sigma_{i}$, we can obtain $\sigma$ as the eigenvalue of matrix $L^{-1} \mathbf{M}$ which gives the criterion for stability of the present system near the equilibrium. That is, if $\sigma_{r}$ is positive the perturbation is unstable, while it is stable for negative $\sigma_{r}$.

\section{Linear problem}

Before discussing the nonlinear problem, we examine the linear response. If we assume a vertically constant zonal wind of Usinly, stationary waves assumed as $\phi \sim e^{i(k x+m z)}$ : satisfy the following dispersion relation.

$$
m^{2}=\frac{B r}{\sin ^{2} \phi_{0}}\left[\frac{\beta}{U \varepsilon}-k^{2}\right]-\frac{1}{4}
$$

where it is noted that the second term in the square bracket on the right is not $k^{2}+l^{2}$, but $k^{2}$ owing to the assumed $y$-dependence of $U_{R}$. For the wave to be able to propagate upward, $\mathrm{m}^{2}$ must be positive. Therefore, $\mathrm{U}$ should lie in the following region (cf. Charney and Drazin, 1961).

$$
0<U<\frac{\beta / \varepsilon}{k^{2}+\frac{1}{4} \frac{\sin ^{2} \phi_{0}}{B r}} \equiv U_{T}
$$

and $U_{T}$ is $56.8 \mathrm{~m} / \mathrm{s}$ in its dimensional magnitude.

The steady linear response of wave in the zonal wind $U_{R}$ given by (8) over the corrugated bottom (6) is obtained by solving the first $2 \mathrm{~N}$ simultaneous linear equations of (16) in which 
$\bar{u}$-terms are omitted and the time tendency terms set to be zero.

The wave amplitudes multiplied by $e^{-z / 2}$ for each $\Lambda$ are shown in Fig. 1(a), while the corresponding $U_{R}$ in Fig. 1(b), in which thick solid line indicates the altitude of turning point where $U=U_{T}$. Above this line the wave becomes external. It is seen from these figures that only in winter time $(\Lambda>0)$ the wave can propagate upward. It is noted that large amplitudes appear along $U=U_{T}$ line. This can be understood by the conservation law of wave action $\partial \widetilde{A} / \partial t+\partial C_{g z} \widetilde{A} /$ $\partial z$, where $\widetilde{A}$ is the wave action and $C_{g z}$ is the group velocity. At the turning point, the group velocity becomes zero, and hence stationary waves $(\partial / \partial t=0)$ have large amplitude near that point. It is further noted that two amplitudes peaks are located around about $40 \mathrm{~km}$ height for $\Lambda=1$ and about $20 \mathrm{~km}$ height for $\Lambda=2$. This could be resulted from resonance.

In Fig. 2, shown are the wave amplitudes with the density factor at 40,55, $70 \mathrm{~km}$ levels for each 1 . We can see again that large amplitudes appear in the zonal wind close to $U_{T}$. Since the altitude of turning point is high (low) for small (large) $\Lambda$ in this model, the value of $\Lambda$ which gives the maximum response becomes small with increasing height.

In closing this section, it is emphasized that the above mentioned response would result from

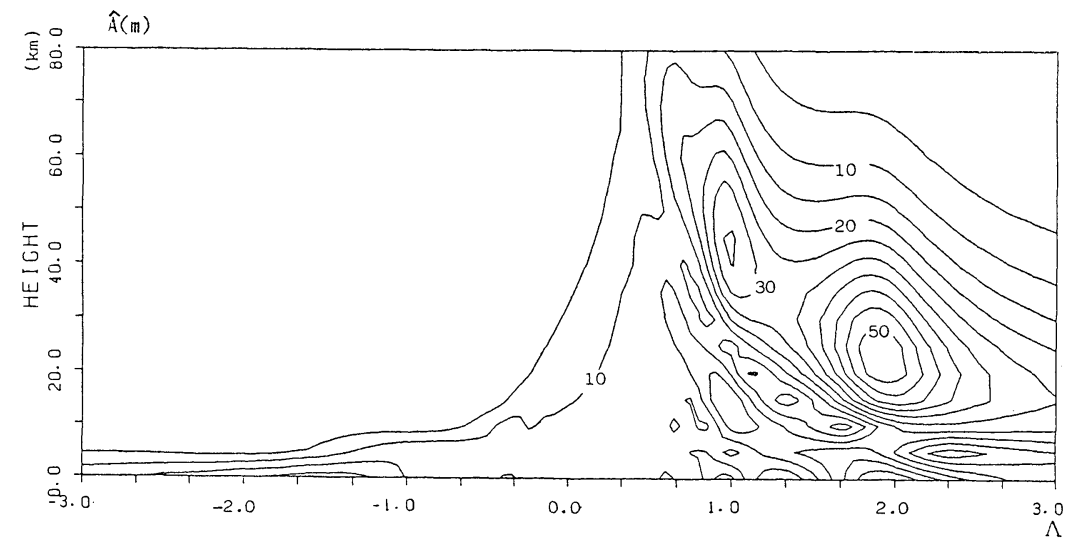

Fig. 1(a). The linear response of wave in the zonal fow $U_{R}$ over the corrugated bottom. The contours are the isopleths of wave amplitude $\hat{\mathrm{A}}\left(=\mathrm{Ae}^{-\mathrm{z} / 2}{ }^{R}\right.$ where $\hat{\mathrm{A}}$ is actual wave amplitude $\left.\left|\Phi^{*}\right| \sin \left(\phi_{0}\right) / g\right)$.

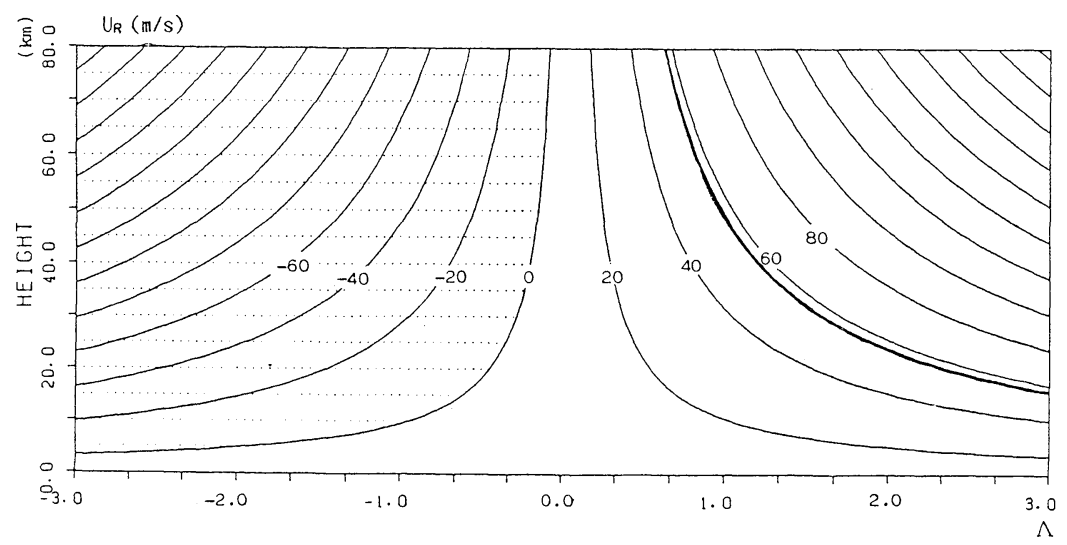

Fig. 1(b). Basic zonal winds $U_{R}^{*}=1 z^{*}+10(\mathrm{~m} / \mathrm{s})$. The thick solid line shows the turning level where $U_{R}=\mathrm{U}_{T}$. 


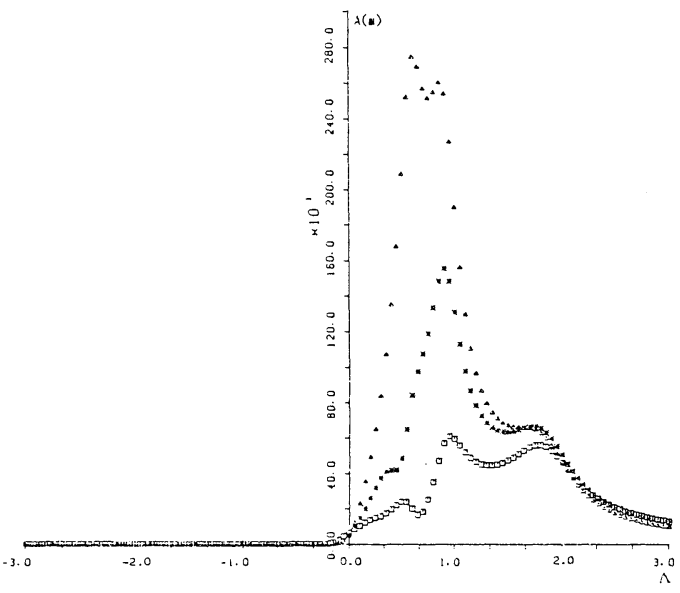

Fig. 2. Wave amplitude $\mathrm{A}$ at three different levels obtained by for the linear problem. Symbols $\square, *$ and $\Delta$ correspond to $z=40,55$ and $70 \mathrm{~km}$, respectively.

the effect of wave propagation near the turning point and partly from the resonance.

\section{Nonlinear equilibrium solutions and their stabilities}

The nonlinear equilibrium solutions can be obtained from (16) with $\partial / \partial t=0$, and their stabilities are examined by the method outlined in section 2 .

Fig. 3 shows the nonlinear responses at 55 $\mathrm{km}$ level for each $A$ : Fig. 3(a) is for the wave amplitude, while 3(b) for the mean zonal flow $U_{R}+\bar{u}$. It is seen that a loop appears in the amplitude response curve and three equilibria are obtained for $1.3<\Lambda<1.6$. The examination of stability gives that branch $\mathrm{A}$ and $C$ are stable, while branch $\mathrm{B}$ is unstable. It is emphasized that we do not have any vacillating solution as obtained by Holton and Mass (ibid) and also by Chao (ibid) (see the last paragraph of this section and Appendix B for further discussions).

As is seen from Fig. 3(a), the wave amplitude on branch $\mathrm{C}$ is larger than that on branch $\mathrm{A}$ near $\Lambda=1.5$ where the response is strong. On the other hand, Fig. 3(b) shows that the corresponding mean zonal flow is a strong westerly flow for the former wave, while it is a weak easterly flow for the latter. These results suggest that the wave on branch $\mathrm{C}$ is essentially external and the wave on branch $\mathrm{A}$ is internal. Fig. 4 which shows

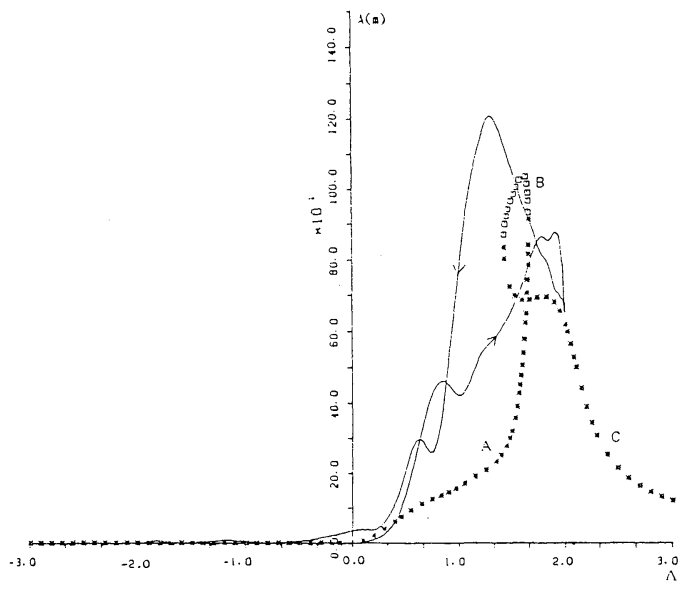

Fig. 3(a). Wave amplitude at $\mathrm{z}=55 \mathrm{~km}$ for the nonlinear problem plotted for each $A . *$ depicts the stable equilibrium solutions and $\square$ the unstable solutions. The solid line shows the trajectory of state obtained by solving the initial value problem discussed in section 6 .

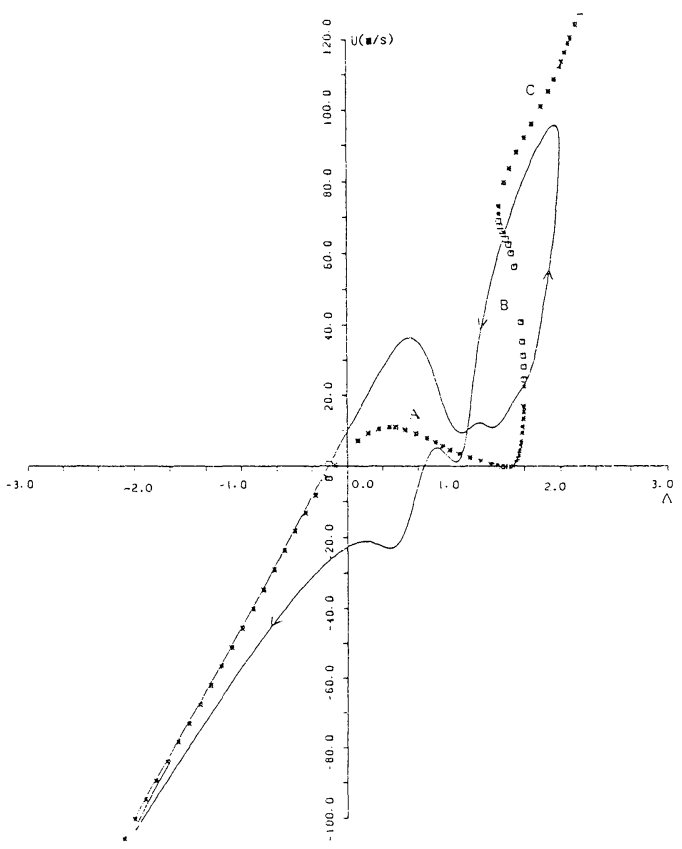

Fig. 3(b). Mean zonal wind $U=U_{R}+\bar{u}$ at $55 \mathrm{~km}$ height, corresponding to Fig. 3(a).

the vertical structures of waves for $A=1.5$ confirms the difference between these two waves. The wave on branch A propagates upward and is absorbed near $55 \mathrm{~km}$ level, where a critical level is formed as the result of easterly accelera- 


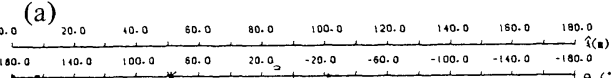

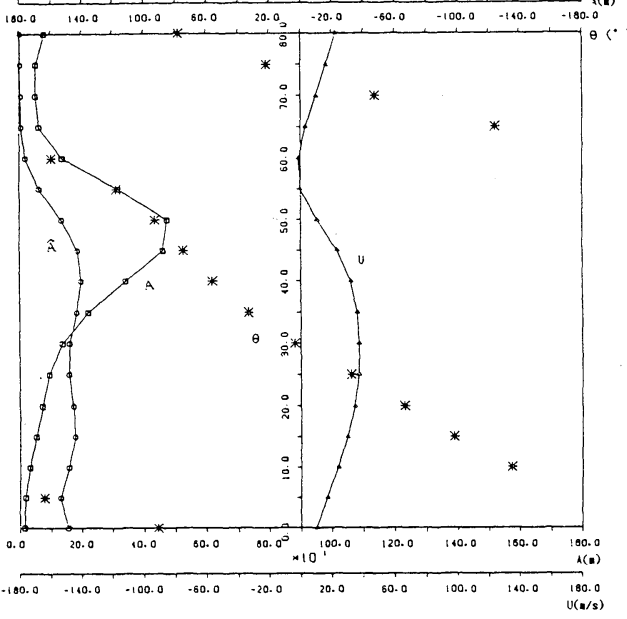

(b)

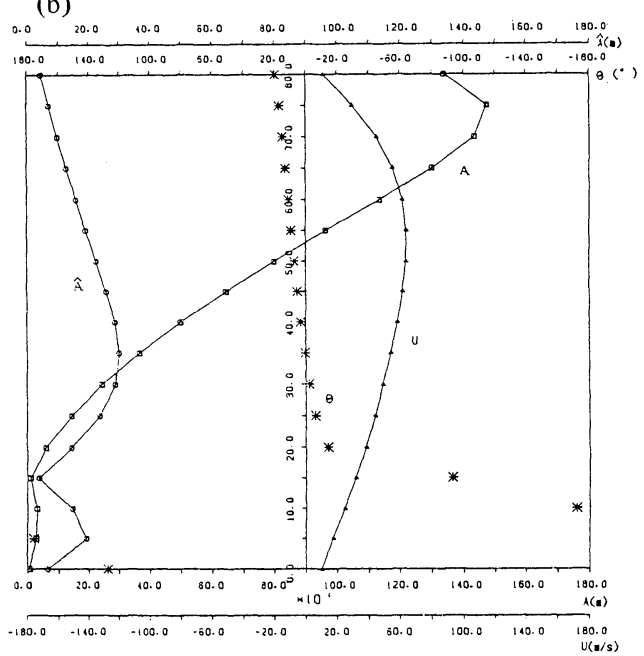

(c)

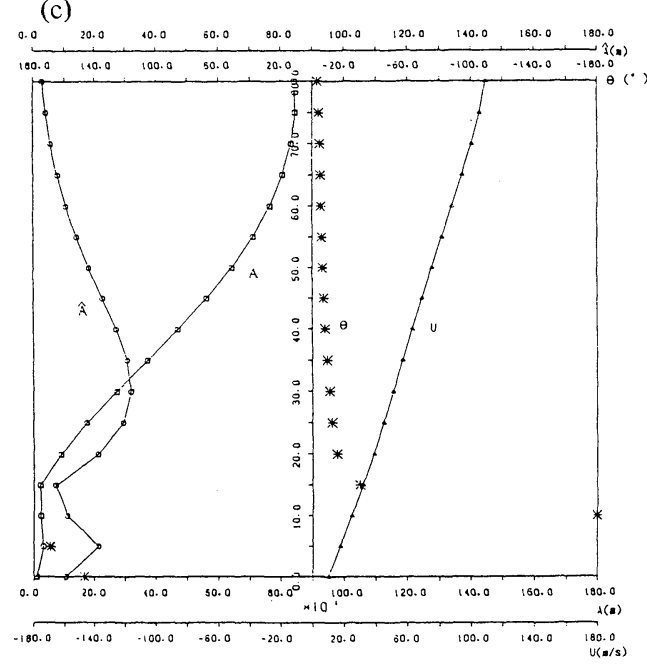

tion of it (Fig. 4(a)). The wave on branch $\mathrm{C}$ is almost external above $20 \mathrm{~km}$ level and does not interact strongly with the basic zonal flow in spite of its large amplitude due to the basic density stratification.

As to the wave on branch $\mathrm{B}$, it is interesting to note that in the background westerly flow whose maximum value is a little smaller than $U_{T}$, it is slightly internal above $25 \mathrm{~km}$ with $m=0$ and grows upward owing to the density stratification.

These structural differences of wave are crucially important to cause the loop in the amplitude response in the present nonlinear system. As is seen in Fig. 1(b), when $\Lambda$ is positive and small, the altitude of turning point is high and hence the wave can be internal almost over the whole layer. Further, in the case of high turning point, the amplitude can be large near it owing to the density stratification and the wave action conservation as already mentioned in section 3. As is well-known, an internal planetary wave can decelerate the basic westerly flow during its upward propagation and feels the refractive index due to the weakened mean zonal flow. Thus, the value of $\Lambda$ which gives the largest response tends to be larger than that in the case of linear response. At $55 \mathrm{~km}$ level, we have the greatest response near $\Lambda=1$ in the linear problem as is shown in Fig. 1(a). However, as is seen in Fig. 3(a), the nonlinear result shows that the largest response on branch $\mathrm{A}$ is near $\Lambda=1.6$ at $55 \mathrm{~km}$ level and the amplitude is rather smaller than that in the linear response. It is noted that the smallness of the wave amplitude is due to the easterly flows formed around this level, i.e., a critical level absorption occurs (Fig. 5).

On the other hand, if $\Lambda$ is positive and large, the turning point is located in lower layers, and the wave is external over deep layers above the point. The basic zonal flow is not almost changed by the wave, in spite of large amplitude in the

Fig. 4(a, b, c). The structures of multiple equilibrium solutions at $\Lambda=1.5$. $\square$ and $O$ show the wave amplitude $\mathrm{A}$ and the amplitude $\hat{\mathrm{A}}$ $\left(=A \mathrm{e}^{-\mathrm{Z} / 2}\right)$, respectively. $*$ shows the phase of wave. $\Delta$ depicts the zonal wind $U$. (a) and (c) are the stable equilibrium solutions, and (b) is the unstable solution. 
upper layers. Then, $\Lambda$ to give the maximum response on branch $\mathrm{C}$ tends to be smaller than that in the case of internal wave, i.e., on branch A, but larger than that in the linear case because of weak interaction with the mean zonal flow. Thus, the loop in the amplitude response curve can be attributed to the structural difference of waves on branches $\mathrm{A}$ and $\mathrm{C}$.

The waves on branch $\mathrm{B}$ are slightly internal and weaken the basic westerly flow to the extent that the maximum velocity does not exceed $U_{T}$. This type of wave can grow upward extremely owing to the density stratification. Thus, the equilibrium including this wave is unstable, because the mean zonal flow can be greatly affected by a small perturbation of wave amplitude or phase.

We add here that the results obtained by a model without the sponge layer above $80 \mathrm{~km}$ is not almost different from the present ones.

As is already known (e.g. Charney and DeVore, 1979; Wakata and Uryu, 1984a, b), the stable solutions on branch $\mathrm{A}$ or $\mathrm{C}$ can be attained asymptotically at time infinity, and it depends on the initial conditions which branch is chosen. The initial value problem with the present model will be discussed in the next section. As has been previously mentioned, we do not have any vacillating solution as obtained by Holton and Mass (ibid) in the parameter region used in the present work (see also Fig. 8). The main reason for this difference could be due to two facts that (i) they and also Chao (ibid) did not give the vertical velocity at the bottom as done in the present paper but gave the geopotential height and (ii) we assume the Ekman damping at the bottom. As is pointed out in Appendix B, the responses of their model are considerably different from ours. It is seen in Figs. $A(a)$ and $A(b)$ that the branch (branch $A$ in our naming) which Cao speculated to be unstable, is certainly unstable for $0.8<A<1.2$ and shows a Hopf bifurcation in which the disturbance grows with oscillation. Furthermore, it is stable for $1.2<\Lambda<1.6$. As to the vacillating solution, we have confirmed that it exists stably, in addition to two stable and one unstable steady solutions, for $1.2<\Lambda<1.6$. It is further noted that there are five equilibrium solutions (two stable and three unstable) near $A=1.6$.

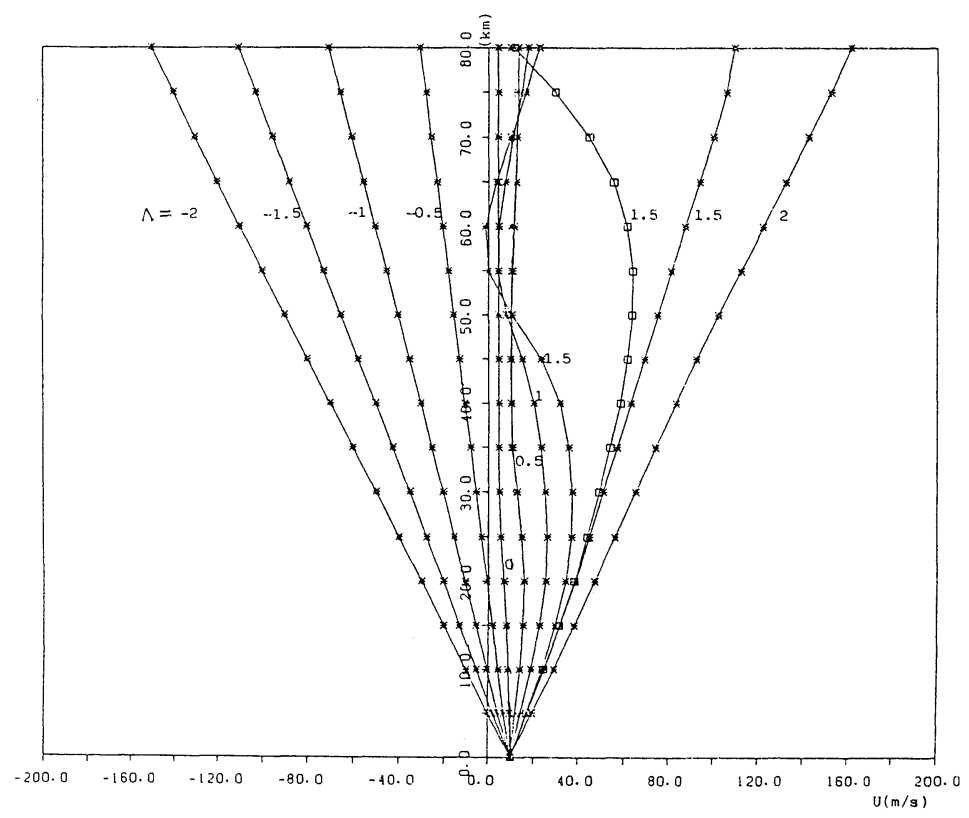

Fig. 5. Height distributions of the mean zonal wind for each $A$. Three equilibrium solutions are obtained at $A=1.5$. $*$ depicts the stable solution and $\square$ the unstable solution. 


\section{Time-dependent problem}

The equilibrium solutions in the preceding section are attained at time infinity. However, the solar differential heating varies in time with one year period, and in this sense the actual atmosphere is never in equilibrium. In the following, we shall investigate the time-dependent behaviors of the present model and interpret the results by comparing with the equilibrium solutions discussed in the previous section.

For doing so, we assume that the basic vertical wind shear $A$ changes with one year period. In other words, we use $\Lambda$ as the indicator of seasonal change of solar heating. The functional form of $\Lambda$ is as follows.

$$
\Lambda=2 \cdot \cos \left(\mathrm{T}^{*} / 365 \text { (days) }\right)
$$

where we assume that $\Lambda=2$ and -2 correspond to the winter and summer solstices, respectively, while $\Lambda=0$ to the eqinoxes. As the initial condition, we adopt the equilibrium solution for $A=$ -2 .

The time-height sections of wave amplitude and the mean zonal flow are shown in Figs. 6(a) and (b), respectively. It is clearly seen that the wave grows twice in a winter, i.e., once in early winter and once in late winter and that the westerly winds become strong between these two peaks of the wave amplitude. We can see also that just after the wave amplification in the late winter, the mean zonal winds change drastically, i.e., much faster than in autumn, from westerly to easterly, and the wave decays as the easterlies descend. This corresponds to the final warming. It is noted that these results are very similar to those obtained from the observational data by Smith (1983), and also those by Holton and Wehrbein's (1979) numerical simulation.

By comparing the present results with the equilibrium solutions why these responses occur. In Fig. 3, we show the trajectories of time-dependent solutions obtained at $55 \mathrm{~km}$ level, together with the equilibrium solutions. Until $A$ comes to near 0.3 , the wave amplitude is almost zero or very small, and the mean zonal flow changes almost exactly along the line of $U_{R}$. That is, the state are on branch A. As $A$ exceeds 0.3 , the wave amplitude begins to grow and attains a small maximum near $\Lambda=0.6$, while the mean zonal flow is still close to the basic flow. It is noted that the both of the trajectories deviate considerably from the steady response curves. This deviation depends on the speed of change of $\Lambda$. When $\Lambda$ exceeds 0.6 , the wavemean flow interaction begins to be strong and the mean zonal flwo becomes weak. Until $A$ reaches about 1.6 at which the greatest response is obtained on branch A in the steady problem, the trajectories are somewhat closer to the steady response curves (branch A). At $\Lambda=1.6$, the state jumps from branch $A$ to $C$. Although the wave seems to change gradually in Fig. 3(a), the mean zonal flow becomes strong with a remarkable acceleration. On branch $\mathrm{C}$, the wave is external over deep layers. It does not almost interact with the mean zonal flow in spite of its large amplitude. Therefore, the mean zonal flow tends to adjust to or recover the basic state. The first amplification of wave in early or mid winter and

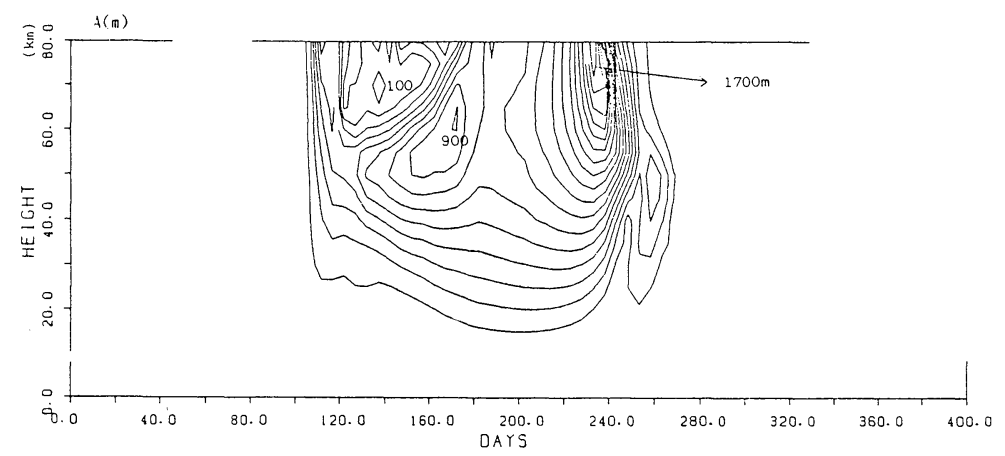

Fig. 6(a). Time-height section of the wave amplitude obtained for initial value problem, with $\Lambda=$ $2 \cos \left(T^{*} / 365\right.$ days). The equilibrium solution at $A=-2$ is adopted for the initial condition. 


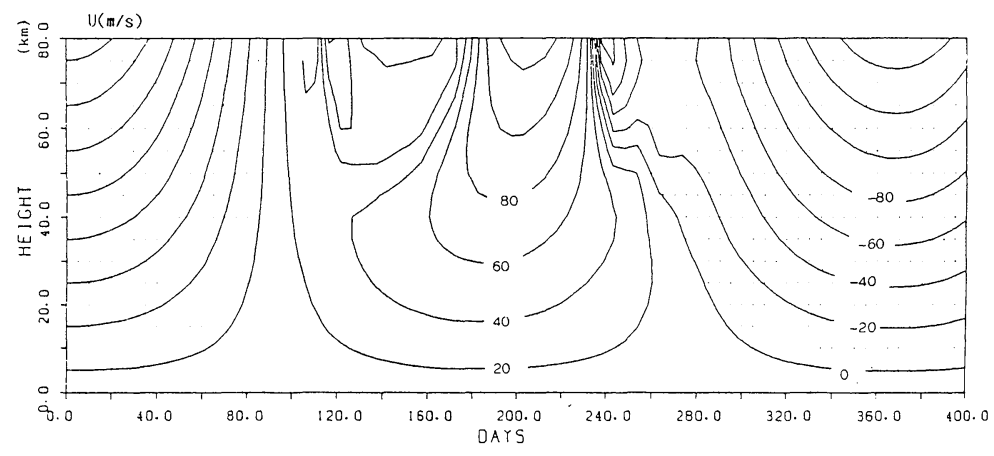

Fig. 6(b). Same as Fig. 6(a) but for the mean zonal winds.

the intensification of the mean zonal flow after that shown in Fig. 6 correspond to this jump.

When $A$ becomes close to 2 (winter solstice), the wave amplitude becomes small, because the westerly in the upper layer becomes strong. As $A$ decreases from 2, the turning point ascends and also the wave-mean flow interaction begins to occur, though weakly. When the speed of the mean zonal flow in the upper stratosphere where nonlinear effects begin to be effective becomes just below the critical velocity $U_{T}$, the wave turns slightly internal with $m=0$ and its amplitude becomes extremely large because of the density stratification and the wave action conservation in the sheared zonal winds near $U_{T}$. The state breaks down toward that on branch A owing to the disappearance of branch C. At the same time, the mean zonal flow is strongly decelerated, being associated with a small overshooting and ultimately changed to the summertype easterlies. The amplification of wave and a drastic appearance of the easterly wind in the late winter or early spring shown in Fig. 6 correspond to this transition of state.

Thus, in the framework of the present model, the final warming can be interpreted as the jump of state from branch $\mathrm{C}$ to branch $\mathrm{A}$. This jump is caused by the decrease of vertical shear of the basic zonal wind corresponding to the change of the solar differential heating. It is noted that our interpretation is not inconsistent with those by Matsuno (1971), Palmer (1981a, b) and so on, because the key mechanism proposed by Matsuno acts also in each one-shot during the jump from branch $\mathrm{C}$ to $\mathrm{A}$ in the present model.

\section{Discussions and conclusions}

Using a simplified vertical one-dimensional model, we have discussed the equilibrium of planetary wave and the mean zonal flow in the stratosphere and its stability. We have used the vertical shear of the basic zonal wind $A$ as the indicator of season. That is, the summer situation is indicated by negative $\Lambda$ 's, while the winter by positive $\Lambda$ 's. $\Lambda=0$ corresponds to the equinoxes.

It is shown that there are two stable equilibria and one unstable equilibrium around late autumn and around late winter or early spring, while one stable state with the mean zonal wind close to the basic state $U_{R}$ is found in midwinter. In addition, solving the initial value problem by changing $\Lambda$ with one year period, we obtained several results similar to those observed in the stratosphere. For example, the growth of planetary wave occurring twice in winter, the intensification of westerly wind between these two peaks of wave amplitude and the drastic change of westerlies to easterlies associated with the final warming. In the framework of the present theory, those stratospheric events can be interpreted as the transitions between two stable states, under the change in the vertical shear of the basic zonal wind corresponding to the seasonal change in the solar heating. The transition is essentially a cusp-type catastrophe.

In Fig. 7, we show schematically how the mean zonal wind changes from winter to spring, in the form of potential map. The minima of the curves correspond to the stable equilibria, while the maxima to the unstable ones. Fig. 7(a) 
(a)
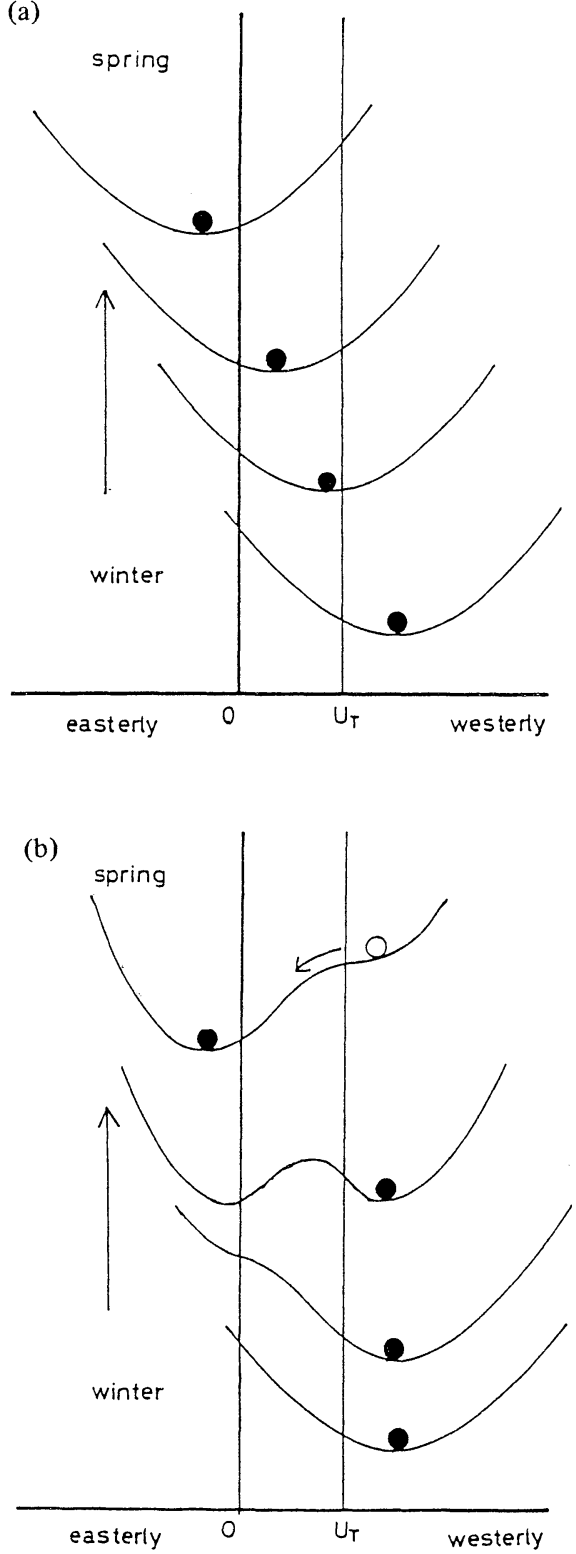

Fig. 7. Schematic potential map for the change from the winter westerly to the spring easterly winds in the stratosphere. The minima of the curves correspond to stable equilibria, while maxima to the unstable equilibrium. Any drastic change does not occur for the slow (i.e., seasonal) change of $A$ in the case of linear response (a), while it occurs in the nonlinear response (b). is the linear case, in which the mean zonal flow changes gradually from westerly to easterly, according as the vertical shear decreases slowly. On the other hand, in the nonlinear case as is shown in Fig. 7(b), the drastic change to the summer-type easterlies occurs just after the disappearance of the winter stable equilibrium with westerlies.

It is noted that our interpretation on the final warming is not inconsistent with many previous works such as Matsuno (1971) and so on, because the key mechanism in Matsuno's model acts also in each one-shot of the event in the present model.

Further, the final warming can be regarded as the instability of polar vortex. It should be, however, emphasized that this instability is closely connected with the disappearance of stable equilibrium solution and the growth rate at the critical point is just zero. Such a disappearance is resulted from the nonlinear interaction of planetary wave and the mean zonal flow. In an equilibrium in late winter, a small change of the refractive index makes the wave propagate easily and the amplitude becomes large particularly in the upper layers. The wave changes, in turn, the refractive index by accelerating easterly flows. Thus, a small deviation from the equilibrium grows through the repetition of such a feedback effect. This instability is completely nonlinear, and different from that in the case of the resonant growth of wave and its breakdown as in Plumb (1981) and so on.

Here, we should compare the present results with Holton and Mass (1976) and Chao (1985). One of the most important differences is that we have no vacillating solution. This is due to the difference in the bottom boundary condition. Chao speculated the existence of multiple solutions in the Holton and Mass model. We have checked it and mention briefly in Appendix B.

As to the external parameters used in our model, we should note that the amplitude of bottom corrugation $\left|h^{*}\right|=100 \mathrm{~m}$ and the meridional temperature difference $\Delta T=10 \Lambda(|\Lambda|$ $\leqq 3$ ) may be a little smaller than those in the actual atmosphere. Since the effect of meridional propagation is not included in the present model, we have assumed these smaller values 
so that the wave amplitude can be reasonable in the stratosphere. If we choose larger values for the external parameters, the region of multiple equilibria expands as is shown in Fig. 8. In their parameter region, the vacillating solution is not present. The model will manifest better the dynamics dicussed in this paper.

Finally, we should note that we have not treated the time variation of the forcing at the bottom. As is seen in Fig. 7(b), the potential valley becomes flat in late winter, and hence the time-dependent solution will become sensitive to the variation of forcing. Further, in the actual situation, the forcing includes many components varying in time. These may explain why the so-called major or minor warming which is not long-lasting occurs in late winter.

Since the present one-dimensional model excludes some important effects such as the meridional propagation of wave, we will try to extend the present work to a spherical model in near future.

\section{Acknowledgement}

This work was partly supported by Japan Society for the Promotion of Science. We would like to express our sincere thanks to the staff members of the dynamics group of Kyushu University. The numerical computations were performed by the use of FACOM M380S computer at Kyushu University.

\section{Appendix A}

In the present paper, the following symbols are used unless otherwise mentioned.

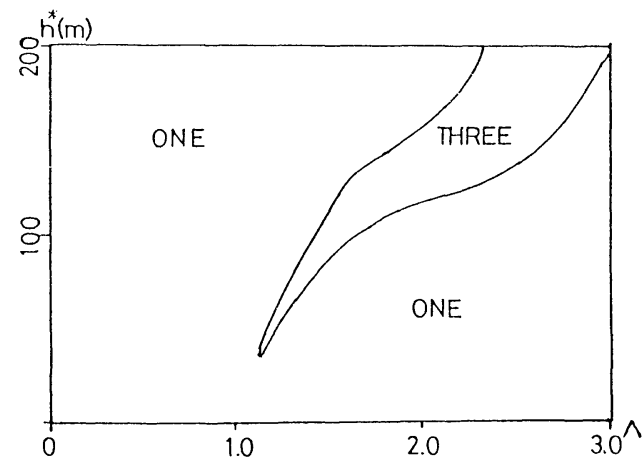

Fig. 8. The existence region of multiple solutions, projected onto for $\mathrm{h}^{*}\left(=2 \mathrm{~h}_{0}\right)$ and $A$ plane.
$L$ : representative horizontal length $\left(=5 * 10^{6}\right.$ $\mathrm{m})$

$H$ : scale-height $\left(\mathrm{RTo} / \mathrm{g}=7 * 10^{3} \mathrm{~m}\right)$

$U$ : representative speed of the basic zonal flow $(=20 \mathrm{~m} / \mathrm{s})$

$g$ : acceleration of garvity $\left(=9.8 \mathrm{~m} / \mathrm{s}^{2}\right)$

$\phi$ : reference latitude of $\beta$-plane $\left(=45^{\circ}\right)$

$\Omega$ : angular velocity of the earth $\left(=0.7 * 10^{-4} /\right.$ s)

$f$ : Coriolis parameter at the reference latitude $\left(=2 \Omega \sin \left(\phi_{0}\right)=0.99 * 10^{-4} / \mathrm{s}\right)$

$a$ : radius of the earth $\left(=6.4 * 10^{3} \mathrm{~km}\right)$

$C_{p}$ : specific heat of dry air at constant pressure $\left(=1004 \mathrm{JK}^{-1} \mathrm{~kg}^{-1}\right)$

$R$ : gas constant for dry air $\left(=287 \mathrm{JK}^{-1} \mathrm{~kg}^{-1}\right)$

$\kappa:$ ratio of $R$ to $C_{p}\left(=R / C_{p}=2 / 7\right)$

$T_{0}:$ a basic temperature which is a function of height only

$N$ : buoyancy frequency squared $\left(=R\left(d T_{0} /\right.\right.$ $\left.\left.d z+k T_{0} / H\right) / H=4 * 10^{-4} \mathrm{~s}^{-2}\right)$

$R_{O}$ : Rossby number $\left(=U / 2 \Omega L=2.86 * 10^{-2}\right)$

$\beta$ : non-dimensional Rossby parameter $\left(=R o^{-1} \cos \left(\phi_{0}\right) L / a^{*}=19.3\right)$

$B_{r}$ : Burger number $\left(=(N H / 2 \Omega L)^{2}=0.04\right)$

$S$ : static stability parameter $\left(=(N H)^{2} / \mathrm{gH}=\right.$ 0.286)

$E$ : Ekman number $\left(=\sqrt{\nu /(2 H f)}\right.$ sin $2 \alpha_{0} \mathrm{Br} /$ $\operatorname{Rosin}\left(\phi_{0}\right)=0.0402$ with $\nu=8 \mathrm{~m}^{2} \mathrm{~s}^{-1}$ and $\alpha_{0}=22.5^{\circ}$, where $\nu$ and $\alpha_{0}$ being eddy viscosity and the angle between isobars and surface wind direction, respectively).

$\alpha_{N}$ : non-dimensional coefficient of Newtonian cooling which is assumed to be a function of height.

$\alpha_{R}$ : non-dimensional coefficient of Rayleigh friction which is assumed to be a function of height.

$t$ : time, scaled by $L / U$

$x, y$ : eastward-, northward-directed Cartesian coordinates, respectively, scaled by $L$.

$z$ : height in log-pressure coordinate, scaled by $H$.

$u, v: x$ and $y$ components of velocity, respectively, scaled by $U$.

$w$ : a measure of vertical velocity, scaled by $H U / L$.

$k$ : vertical unit vector

$\nabla$ : horizontal gradient vector

$k$ : non-dimensional zonal wave number $(=2.21)$ 
$l$ : non-dimensional meridional wave number $(=\pi)$

$\phi$ : transformed geopotential, scaled by $2 \Omega$ $U L$, which is related to geopotential $\Psi$ by $\Psi=\sin \left(\phi_{0}\right) e^{z / 2} \Phi$

$T$ : temperature, scaled by $2 \Omega U L / R$.

$h$ : amplitude of the bottom corrugation, scaled by $H$.

\section{Appendix B}

\section{Comments on Holton and Mass' or Chao's model}

Holton and Mass (1976) studied the behaviors of planetary wave in the stratosphere by using a one-dimensional model. As the bottom boundary condition, they gave geopotential instead of vertical velocity. They found the vacillating solution for the forcing larger than some critical value. Later, Holton and Dunkerton (1978) showed that either steady solution or vacillating solution appears, depending on the initial condition. Recently, Chao (1985) has re-examined the results of Holton and his colleagues above, in the light of the concept of catastrophe. By solving the initial value problems for several amplitudes of the bottom forcing, he has checked the critical point of transition from steady to vacillating solutions, and made a diagram to show the existence region of multiple equilibria, which is similar to Fig. 8. However, as his paper remains conceptual and conjectural in many aspects, we have examined the behaviors of his or Holton and Mass' model on the basis of the present method. We have used the following numerical values: $k^{*}=2 / a_{*} \cos (\pi / 3), l^{*}=3 / a^{*}$ and $\Delta z=5 \mathrm{~km}$. The bottom geopotential has been given as $\left|\phi^{*}\right|$ $\sin (\pi / 3) / g=25 \mathrm{~m}$ at $z^{*}=10 \mathrm{~km}$.

In Figs. $A(a)$ and $A(b)$, shown are the responses of wave amplitude at $55 \mathrm{~km} \mathrm{level} \mathrm{and}$ the corresponding mean zonal flow, respectively. It is seen from these figures that the responses are considerably different from those discussed in this paper. The wave amplitude response curve has a double loop and there exist five equilibium solutions around $\Lambda=1.6$. It should be emphasized that the branch (branch $\mathrm{A}$ in our naming) which Chao speculated to be unstable is certainly unstable for $\Lambda(0.7<\Lambda<1.2)$, where there is seen a Hopf bifurcation in which disturbance grows with oscillation. But it is stable

\section{for $1.2<\Lambda 1.7$.}

Solving the initial value problem by increasing $\Lambda$, we have confirmed that there are stably vacillating solutions for $1.2<\Lambda<1.7$ where two stable steady and one unstable steady solutions exist. We note here that it is impossible to find stable equilibriums solutions on branch $\mathrm{A}$ in $1.2<\Lambda<1.7$ by such a method as Chao's. By trac-

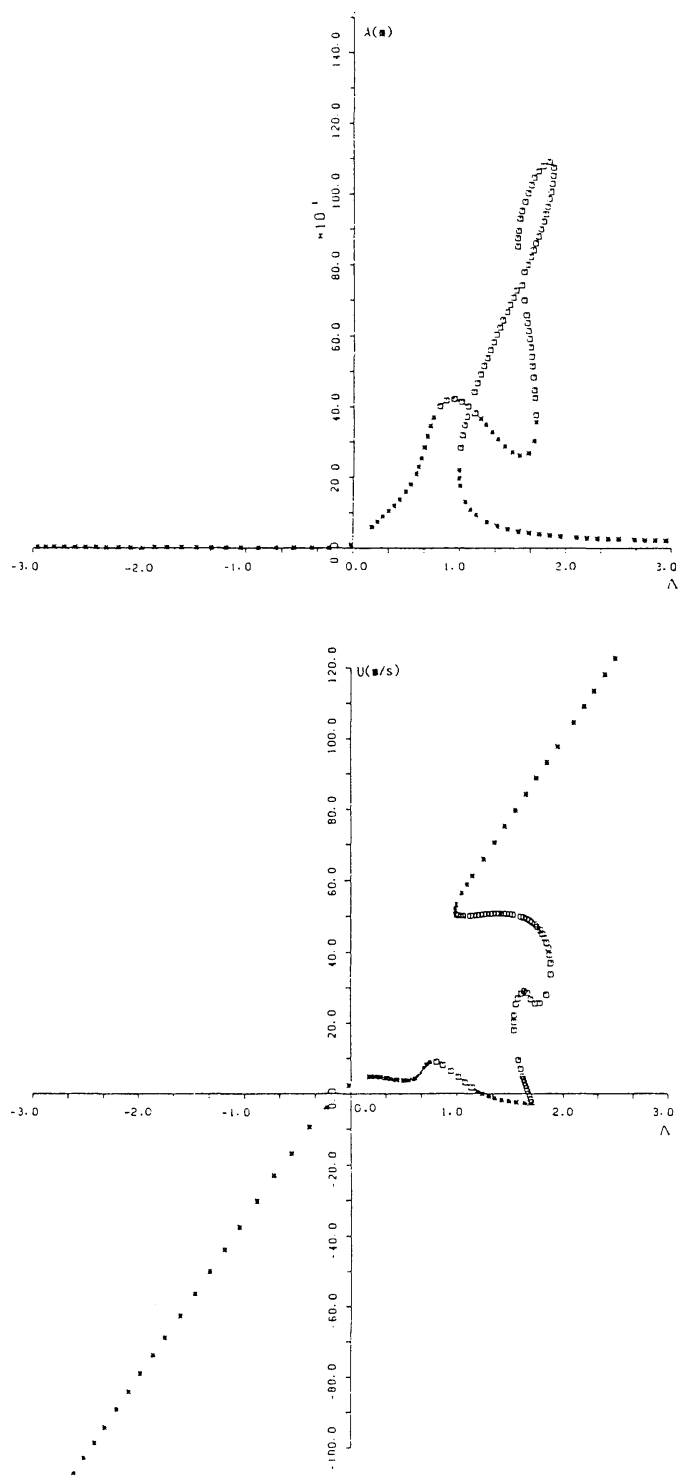

Fig. 9. Same as Fig. 3 but for Holton and Mass' or (Chao's) model. The bottom boundary condition: $\left|\Phi^{*}\right| \sin (\pi / 3) / \mathrm{g}=25 \mathrm{~m}$ at $10 \mathrm{~km}$ altitude (the density factor is excluded). 
ing the time evolution of perturbations around the stable steady state solution, we have also confirmed that the occurrence of vacillating solution depends on the magnitude of initial perturbations. That is, if the perturbation is sufficiently small, the solution tends to settle down to the stable steady state, while we have a vacillation if the perturbations large. It has been further confirmed that the vacillating solution is readily attained if we start from the unstable solution on branch A near $\Lambda=0.7$. These are consistent with the conclusion of Holton and Dunkerton (1978).

As has been mentioned in the text, we have no vacillating regime for the range of parameter used in our model. Our solution settles down to an equilibrium. The summer-type 'permanent' easterly wind is attained after the final warming. It never oscillates as in the actural atmosphere. The difference of our results from Chao's seems to be due to that in the bottom boundary condition. We emphasize that as the bottom boundary condition for a bottom forcing problem, $w$-condition could be better than $\Phi$-condition. For the latter condition cannot express appropriately the free mode with $w=0$ at the bottom and hence the barotropic mode resonance.

\section{References}

Chao, W.C., 1985: Sudden stratospheric warmings as catastrophes. J. Atmos. Sci., 42, 1631-1646.

Charney, J.G, and J.G. DeVore, 1979: Multiple flow equilibria in the atmosphere and blocking. J. Atmos. Sci., 36, 1205-1216.

and P.G. Drazin, 1961: Propagation of planetary-scale disturbances from the lower into the upper atmosphere. J. Geophys. Res., 66, 83-109.

Clark, J.H.E., 1974: Atmopsheric response to the quasiresonant growth of forced planetary waves. $J$. Meteor. Soc. Japan, 52, 143-163.

Geisler, J.E., 1974: A numerical model of the sudden stratospheric warming mechanism. J. Geophys. Res.,
79, 4989-4999.

Holton, J.R., 1975: The dynamic meteorology of the stratosphere and mesosphere. Meteor. Monogr., No. 37, Amer. Meteor. Soc., 218 pp.

and C. Mass, 1976: Stratospheric vacillation cycles. J. Atmos. Sci., 33, 2218-2225.

- and T.J. Dunkerton, 1978: On the role of wave transience and dissipation in stratospheric mean flow vacillations. J. Atmos. Sci., 35, 740-744.

- and W.M. Wehrbein, 1980: The role of forced planetary in the annual cycle of the zonal mean circulation of hte middle atmosphere. J. Atmos. Sci., 37, 1968-1983

Kanzawa, H., 1982: Eliassen-Palm flux diagnostics and the effect of the mean wind on planetary wave propagation for an observed sudden stratospheric warming. J. Meteor. Soc. Japan, 60, 1063-1073.

Lighthill, J., 1978: Waves in fluids. Cambridge University Press, $504 \mathrm{pp}$.

O'Neill, A. and C.E. Youngblut, 1982: Stratospheric warmings diagnosed using the transformed Eulerianmean equations and the effect of the mean state on wave propagation. J. Atmos. Soc., 39, 1370-1386.

Palmer, T.N., 1981a: Diagnostic study of a wavenumber-2 stratospheric sudden warming in a transformed Eulerian-mean formalism. J. Atmos. Sci., 38, 844855.

- 1981b: Aspects of stratospheric sudden warmings studied from a transformed Eulerian-mean viewpoint. J. Geophys. Res., 86, 9679-9687.

Plumb, R.A., 1981: Instability of the distorted polar night vortex: A theory of stratospheric warmings. J. Atmos. Sci., 38, 2514-2531.

Smith, A.K., 1983: Stationary waves in the winter stratosphere: seasonal and interannual variability. J. Atmos. Sci., 40, 245-261.

Tung, K.K. and R.S. Lindzen, 1979a: A theory of stationary long waves. Part I: A simple theory of blocking. Mon. Wea. Rev., 107, 714-734.

and - 1979b: Resonant Rossby waves in the presence of realistic vertical shear. Mon. Wea. Rev., 107, 735-750.

Wakata, Y. and M. Uryu, 1984a: Quasi-resonant Rossby wave. Part I: External wave. J. Meteor. Soc. Japan, $62,388-412$.

and - 1984b: Nonlinear behaviors of forced baroclinic wave in a continuous zonal flow. J. Meteor. Soc. Japan, 62, 809-832. 


\section{成層圈多重平衡解と季節变化}

\section{和 方 吉 信・瓜生道也}

(九州大学理学部物理学教公)

成層圈の平均帯状風と地表の凹凸により強制された感星波の平衡解を鉛直一次元モデルを用いて求め, その安定性を調べた。

晚秋と初春には二つの安定平衡解と一つの不安定平衡解があるが，真冬には一つの安定平衡解になる 事が示される。このモデルでは, Holton and Mass（1976）により得られた振動解は現れない。

次に，基本平均帯状流の鉛直シア一を季節変化の指標として一年の周期で変え，初期值問題を解いた。 冬期に二度の惑星波の增幅とそのピークの間で西風の強化が見られた。さらに、晚冬から初春にかけて 最終昇溫に似た帯状風の西風から東風への急激な変化が見られる。これらの結果は、S mith (1983)の解 析や Holton and Werbein (1980) の数值計算の結果と良く合っている。成層圈風系の主な季節变化は多 重平衡解があるため太陽放射過熱の年変化に伴い起きる履歷現象として解釈できる。

最終舁温を伴う極夜消の崩壊はある種の非線形不安定である。すなわち，もし Charney-Drazin の臨 界速度に近い冬の上部成層圈の西風が，惑星波によって乱されると波の屈折率が変化し，この変化が波 の增幅を引き起こす。増幅した波は再び東風加速を起こし屈折率を変える。この過程が繰り这し起き， ついには極夜渦は壊れる。この不安定はPlumb（1981）らにより議論された共沜による不安定とは異な る。 